\title{
Wetting effect and morphological stability in growth of short-period strained multilayers
}

\author{
Zhi-Feng Huang * Daniel Kandel $\dagger$ and Rashmi C. Desa $\ddagger$ \\ Department of Physics, University of Toronto, Toronto, Ontario, Canada M5S 1A7
}

(Dated: Submitted to Appl. Phys. Lett. 24 January 2003; accepted 1 May 2003)

\begin{abstract}
We explore the morphological stability during the growth of strained multilayer structures in a dynamical model which describes the coupling of elastic fields, wetting effect, and deposition process. We quantitatively show the significant influence of the wetting effect on the stability properties, in particular for short-period multilayers. Our results are qualitatively similar to recent experimental observations in $\mathrm{AlAs} / \mathrm{InAs} / \mathrm{InP}(001)$ system. We also give predictions for strainbalanced multilayers.
\end{abstract}

Strained periodic multilayer films composed of different material layers have attracted much attention since such material structures can have tunable electronic properties. Among the actively investigated systems is the multilayer with alternating tensile/compressive layers coherently grown on a substrate, resulting in a modulated structure like short-period superlattice [e.g., GaAs/InAs 1 or $\mathrm{AlAs} / \mathrm{InAs}$ 2.3.4 on $\mathrm{InP}(001)$ ] or a multiple quantum well [e.g., $\mathrm{GaInP} / \mathrm{InAsP}$ on $\mathrm{InP}(001)]^{5}$. Generally morphological instability occurs in such coherent lattice-mismatch multilayer structures, driven by a gradual release of misfit-generated stresses when dislocations are absent. Consequently, undulations are observed in the strained layers, instead of ideally flat interfaces. This stress-driven morphological instability

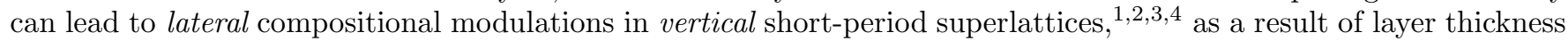
modulations (caused by interface rippling) and different material components in adjacent layers $\stackrel{3.5}{=}$ The spontaneous formation of these lateral modulations is also a promising way to self-organize fabrication of low-dimensional quantum heterostructures, especially quantum wires $\underline{1.2 .3}$

Although understanding of the detailed growth mechanism in multilayer structures is important and of much interest for both fundamental studies and device applications, it is still far from complete due to the complexity of the system. This complexity arises from the coupling of strain fields in different layers, and the nonequilibrium nature of the growing film for which material deposition rates play an important role in the pattern formation. Previous theoretical analyses use some approximations to determine the elastic fields, treating each buried island ${ }^{6}$ or nonplanar interface ${ }^{7}$ of the multilayer as a misfitting inclusion in a semi-infinite homogeneous medium. Recently, a general method has been developed to directly calculate the elastic state of the multilayer system $\underline{\underline{8}}$

Wetting effects have been considered in the context of single-layer strained film growth $\stackrel{9.10}{\rightleftharpoons}$ but not for multilayers. In this letter, we attempt to remedy this. The wetting effect arises from the change of material properties across interlayer interfaces within the system, and is especially important for thin composite layers. Here, we incorporate the wetting effect (arising from nonlinear elastic contributions ${ }^{10}$ ) in the early morphological evolution of growing multilayer films. We focus on the stability properties of the system and on comparison with recent experiments.

We consider a three-dimensional coherent multilayer structure with $k$ stacking layers deposited on a semi-infinite substrate. In a general case, each composite layer $i(1 \leq i<k)$ could consist of a material different from all the other layers and substrate, with average thickness $l_{i}$ and height profile $z=h_{i}(x, y)=\sum_{j=1}^{i} l_{j}+\sum_{\mathbf{q}} \hat{h}_{i}(\mathbf{q}) \exp \left(i q_{x} x+i q_{y} y\right)$. The film/substrate interface is described by $\hat{h}_{0}(\mathbf{q})$, and the top strained layer $k$ by a surface morphological perturbation $\hat{h}_{k}(\mathbf{q}, t)$ and by an average thickness $l_{k}=v_{k} t$ where $v_{k}$ is the deposition rate.

The basic state of the system is chosen to be a uniform film of flat growing surface and planar underlying interfaces, with all strains zero except for $\bar{u}_{z z, i}=\bar{u}_{i}=\epsilon_{i}\left(1+\nu_{i}\right) /\left(1-\nu_{i}\right)$ due to Poisson relaxation in the $z$ direction. $\epsilon_{i}$ and $\nu_{i}$ are the misfit and Poisson ratio of layer $i$, respectively. The elastic free energy of the multilayer is written as $\mathcal{F}_{\text {el }}=\int_{-\infty}^{h_{0}} d^{3} r \mathcal{E}_{0}+\sum_{i=1}^{k} \int_{h_{i-1}}^{h_{i}} d^{3} r \mathcal{E}_{i}$, where the elastic energy density for each layer $i$ can be generally expressed as $\mathcal{E}_{i}=\overline{\mathcal{E}}_{i}+\delta \mathcal{E}_{i}$. Here $\overline{\mathcal{E}}_{i}$ is the energy density of the basic state and $\delta \mathcal{E}_{i}$ is the change in energy density due to a perturbation. Each of the two terms includes a linear contribution, obtained from linear elasticity, and a nonlinear contribution. The latter is often ignored, but is crucial for the wetting effect 10 It leads to the dependence of $\overline{\mathcal{E}}_{i}$ on layer thickness $h_{i}(x, y)-h_{i-1}(x, y)$. This dependence is attributed to the coupling between the elastic relaxation of layer $i$ and that of the underlying layer or substrate $i-1$. It was shown ${ }^{10}$ that the thickness dependence of $\overline{\mathcal{E}}_{i}$ enhances the wetting of the substrate by the film in the case of a single layer, and consequently stabilizes the flat surface.

For the energy density of the perturbed state, $\delta \mathcal{E}_{i}$, we only keep the linear elasticity contribution $\delta \mathcal{E}_{i}^{\text {lin }}=\bar{\sigma}_{\alpha \beta, i} \delta u_{\alpha \beta, i}+$ $\lambda_{\alpha \beta \xi \rho} \delta u_{\alpha \beta, i} \delta u_{\xi \rho, i} / 2$ (with $\sigma_{\alpha \beta, i}$ the stress tensor and $\lambda_{\alpha \beta \xi \rho}$ the elastic modulus tensor), since the nonlinear contribution is of higher order in the perturbed strains $\delta u_{\alpha \beta, i}$. Thus, we have $\mathcal{E}_{i}=\overline{\mathcal{E}}_{i}+\delta \mathcal{E}_{i}^{\text {lin }}$. Using the results of Ref. 8 for the 
first-order surface elastic energy density, $\delta \hat{\mathcal{E}}_{k}^{\text {lin }}$, we obtain the first-order evolution equation for $\hat{h}_{k}(\mathbf{q}, t)$ :

$$
\begin{aligned}
\partial \hat{h}_{k} / \partial t & =\left\{\sigma_{k}-\Gamma_{k} q^{2}\left[d^{2} f_{\mathrm{el}, k}^{(0)} / d h_{k}^{2}\right]_{h_{k}=\bar{h}_{k}(t)}\right\} \hat{h}_{k} \\
& -\Gamma_{k} E^{\prime} \epsilon_{k} q^{3} e^{-q v_{k} t}\left[\sum_{j=1}^{k-1}\left(\epsilon_{j+1}-\epsilon_{j}\right) e^{-q \sum_{i=j+1}^{k-1} l_{i}} \hat{h}_{j}+\epsilon_{1} e^{-q \sum_{i=1}^{k-1} l_{i}} \hat{h}_{0}\right]
\end{aligned}
$$

with $\sigma_{k}=\Gamma_{k}\left[E^{\prime} \epsilon_{k}^{2} q^{3}-\tilde{\gamma}_{k}(0) q^{4}\right]$ and $f_{\mathrm{el}, i}^{(0)}\left(h_{i-1}, h_{i}\right)=\int_{h_{i-1}}^{h_{i}} d z \overline{\mathcal{E}}_{i}\left(h_{i}-h_{i-1}, z\right)$. The derivatives of the latter quantity with respect to $h_{i}$ are taken with fixed $h_{i-1}$. Here we assumed that surface diffusion is the dominant mass transport process, with negligible bulk and interlayer diffusion as well as frozen buried interfaces $\stackrel{8}{-}$ We used a step-function form for surface stiffness $(\tilde{\gamma})$ and misfit stresses $\left(\bar{\sigma}_{x x, i}=\bar{\sigma}_{y y, i}\right)$ at the interlayer interfaces $\stackrel{10}{\underline{10}}$ In Eq. (11) $\Gamma_{k}$ is the surface mobility and $\tilde{\gamma}_{k}(0)$ is the surface stiffness at the orientation of the unperturbed surface. The average surface position is $\bar{h}_{k}=\sum_{i=1}^{k-1} l_{i}+v_{k} t$, and $E^{\prime}=2 E(1+\nu) /(1-\nu)$ with Young's modulus $E$ and Poisson ratio $\nu$ assumed to be identical for all the layers and substrate. Note that the corresponding results for single-layer film can be recovered from Eq. (11) with $k=1$.

The evolution Eq. (1) is similar to that without the wetting effect, $\frac{8}{\underline{3}}$ showing explicitly the influence of interface morphologies and elastic properties of different buried layers on the top surface profile. The important results of this work arise from the term $d^{2} f_{\mathrm{el}, k}^{(0)} / d h_{k}^{2}$, which represents the wetting effect. The exact solution of Eq. (11) has a form similar to the solution in the absence of the wetting effect $\frac{8}{\underline{*}}$ The surface profile $\hat{h}_{k}$ can be expressed in terms of the product of $(2 \times 2)$ characteristic matrices $\overline{\mathbf{L}}_{j}(3 \leq j \leq k)$ as well as the profiles $\hat{h}_{1}$ and $\hat{h}_{2}$ of first and second deposited layers. The elements of the bottom row of $\overline{\mathbf{L}}_{j}$ are 1 and 0 and those of the top row are $\bar{L}_{j, 1}(q)$ and $\bar{L}_{j, 2}(q)$, where

$$
\begin{aligned}
& \bar{L}_{j, 1}(q)=\mu_{j}\left(q, l_{j} / v_{j}\right)\left[1-\Gamma_{j} E^{\prime} q^{3} \epsilon_{j}\left(\epsilon_{j}-\epsilon_{j-1}\right) G_{j}\left(q, l_{j} / v_{j}\right)\right]-\bar{L}_{j, 2}(q) / \mu_{j-1}\left(q, l_{j-1} / v_{j-1}\right), \\
& \bar{L}_{j, 2}(q)=-\mu_{j}\left(q, l_{j} / v_{j}\right) e^{-q l_{j-1}}\left[\Gamma_{j} \epsilon_{j} G_{j}\left(q, l_{j} / v_{j}\right)\right] /\left[\Gamma_{j-1} \epsilon_{j-1} G_{j-1}\left(q, l_{j-1} / v_{j-1}\right)\right] .
\end{aligned}
$$

Here

$$
\begin{aligned}
& \mu_{j}\left(q, l_{j} / v_{j}\right)=e^{\sigma_{j} l_{j} / v_{j}-\Gamma_{j} q^{2} \int_{0}^{l_{j} / v_{j}} d t^{\prime}\left[d^{2} f_{\mathrm{el}, j}^{(0)} / d h_{j}^{2}\right]_{l_{j}=v_{j} t^{\prime}},} \\
& G_{j}\left(q, l_{j} / v_{j}\right)=\int_{0}^{l_{j} / v_{j}} d t^{\prime} e^{-\left(q v_{j}+\sigma_{j}\right) t^{\prime}+\Gamma_{j} q^{2} \int_{0}^{t^{\prime}} d t^{\prime \prime}\left[d^{2} f_{\mathrm{el}, j}^{(0)} / d h_{j}^{2}\right]_{l_{j}=v_{j} t^{\prime \prime}}}
\end{aligned}
$$

In the following these general results, which can apply to both periodic and nonperiodic systems, are used to determine the morphological stability of periodic tensile/compressive multilayer structures, and in particular shortperiod superlattices $\stackrel{1.2 .3 .4}{=}$ In this case the multilayer film is composed of alternating $A$ and $B$ layers, with their associated parameters such as nonzero misfits $\epsilon_{A(B)}^{*}$, layer thicknesses $l_{A(B)}^{*}$, surface mobilities $\Gamma_{A(B)}^{*}$, deposition rates $v_{A(B)}^{*}$ and surface stiffnesses $\gamma_{A(B)}^{*}$. Here we have expressed all the parameters in dimensionless form: $l_{A}^{0}=$ $\tilde{\gamma}_{A}(0) /\left(E^{\prime} \epsilon_{A}^{2}\right)$ and $v_{A}^{0}=\Gamma_{A} E^{\prime 3} \epsilon_{A}^{6} / \tilde{\gamma}_{A}^{2}(0)$, as well as $\epsilon_{B}^{*}=\epsilon_{B} / \epsilon_{A}, \Gamma_{B}^{*}=\Gamma_{B} / \Gamma_{A}$ and $\gamma_{B}^{*}=\tilde{\gamma}_{B}(0) / \tilde{\gamma}_{A}(0)$. To evaluate the wetting effect, we assume the form $d f_{\mathrm{el}, j}^{(0)} / d h_{j}=\overline{\mathcal{E}}_{j}^{\operatorname{lin}}\left\{1-\chi_{j} \exp \left[-\left(h_{j}-h_{j-1}\right) / h_{\mathrm{ML}, j}\right]\right\}$ (for $\left.h_{j}>h_{j-1}\right)$ appropriate for the single-layer case, (according to the qualitative modeling calculations in ref. 10, which yield this general functional form of wetting elastic energy for various lattice structures with diagonal bonds), where $\overline{\mathcal{E}}_{j}^{\text {lin }}=E \epsilon_{j}^{2} /(1-\nu)$ is the linear elastic energy density of the basic state and $h_{\mathrm{ML}, j}$ is the $1 \mathrm{ML}$ thickness of layer $j(A$ or $B$ ). We also assume $\chi_{j}=\chi=0.05$ for all strained layers.

For this periodic $A / B$ system, the characteristic matrices $\overline{\mathbf{L}}_{j}$ presented in Eq. (2) are reduced to two types, $\overline{\mathbf{L}}_{A(B)}$, and the effective stability (for large number of grown layers $k \gg 1$ ) is determined by the maximal magnitude of the eigenvalues $\lambda$ of the matrix $\overline{\mathbf{L}}_{B} \overline{\mathbf{L}}_{A}$, as shown in Ref. 8. When $\max (|\lambda|)>1$, the initial perturbations are amplified after further deposition, leading to a morphological instability; otherwise, the perturbations decay during the growth, leading to a stable flat film profile.

Consequently, the stability diagrams for various growth and material parameters can be determined, as presented in Fig. [1] where in order to compare with experiments, the parameters are chosen to qualitatively represent those of the growth of AlAs/InAs/InP(001) short-period superlattices ${ }^{3.4}$ Note that the global strain of the whole multilayer

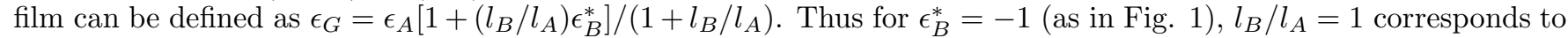
$\epsilon_{G}=0$, while $l_{B} / l_{A}>1$ or $<1$ to tensile or compressive films. Similar to previous calculations without the wetting

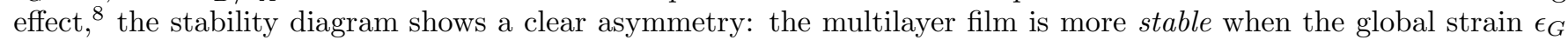
has the same sign as the misfit of the layers with smaller surface mobility. For the system of AlAs/InAs/InP(001), $\Gamma_{\text {InAs }}\left(\Gamma_{A}\right) \gg \Gamma_{\mathrm{AlAs}}\left(\Gamma_{B}\right)$, and then the globally tensile film $\left(\epsilon_{G}<0\right)$ is more stable since $\epsilon_{\mathrm{AlAs}}<0$, as seen in Fig. 
1(a) and in experiments. Furthermore, as shown in Fig. 1(a), without the wetting effect (dashed line), the flat film is stable only when the global strain is tensile. The situation is qualitatively different when the wetting effect is included [solid line in Fig. 1(a)]: both the globally tensile and compressive flat films can be stabilized, although the asymmetry in the stability diagram is still obvious.

The earlier result with the consideration of the wetting effect is qualitatively similar to experimental observations 3.4 in the following way: We plot [Fig. [(b)] $\max (|\lambda|)$ as a function of global strain, $\epsilon_{G}$, for $v_{A}^{*}=0.05$, which corresponds to the horizontal dot-dashed line in Fig. 1(a). To evaluate $\epsilon_{G}$, we set $\epsilon_{A}=3.2 \%$ according to the InAs/InP misfit. As explained earlier, when the maximal value of $\lambda$ is equal to 1 , the multilayer film is stable, with a smooth surface; when it is larger than 1 , the film is unstable, leading to a rough surface. Also, the larger the value of $\max (|\lambda|)$, the rougher the surface that results, as obtained qualitatively from our theoretical results $\hat{h}_{k} \propto|\lambda|^{k / 2}$ for $k \gg 1$. Thus, this figure can be compared with the recent rms roughness measurement of Norman et al. ${ }^{4}$ where the surface is flat or with small roughness for samples under global tension or small compression, but shows a rapidly increasing degree of roughness for larger compression. This experimental finding can be qualitatively inferred from our result including wetting effect [solid line in Fig. 1(b)], but not from the result without wetting (dashed line).

We also find that with the wetting effect, the stability boundary value of $l_{B} / l_{A}$, above which stabilization is possible, depends sensitively on $l_{A}^{*}+l_{B}^{*}$, but only very slightly on $\Gamma_{B}^{*}$. The stability boundary value is closer to 1 (the value in the absence of the wetting effect) when $l_{A}^{*}+l_{B}^{*}$ is larger, showing that the wetting effect has less influence for thicker layer periods.

Since some multilayer systems are grown under strain-balance conditions $\left(\epsilon_{G}=0\right), \underline{\underline{5}}$ it is interesting to study the corresponding stability properties. The diagrams of $l_{B}^{*} / l_{A}^{*}\left(=-1 / \epsilon_{B}^{*}\right.$ for $\left.\epsilon_{G}=0\right)$ versus $v_{A}^{*}$ are shown in Fig. 22 where the stability asymmetry remains and is independent of relative mobility $\Gamma_{B}^{*}$, indicating more stability for asymmetric

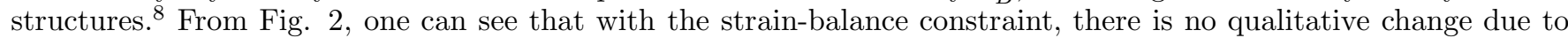
the wetting effect. Also, for short period (e.g., $\left.l_{A}^{*}+l_{B}^{*}=0.3\right)$ and large mobility difference (e.g., $\left.\Gamma_{B}^{*}=0.001\right)$, the wetting effect tends to render the film more stable.

In summary, we have studied the interplay of elasticity, wetting layer effects and material deposition in coherent multilayer growth, and determined the stability and morphological evolution of growing films. The important influence of the wetting effect for short-period multilayers has been calculated, reproducing recent experimental measurements. We hope these results will be helpful for practical growth of low-dimensional nanostructures.

* Electronic address: huang@csit.fsu.edu Present address: School of Computational Science and Information Technology, Florida State University, Tallahassee, Florida 32306-4120

$\dagger$ Electronic address: daniel.kandel@weizmann.ac.il Permanent address: Department of Physics of Complex Systems, Weizmann Institute of Science, Rehovot 76100, Israel

\# Electronic address: desai@physics.utoronto.ca

1 K. Y. Cheng, K. C. Hsieh, and J. N. Baillargeon, Appl. Phys. Lett. 60, 2892 (1992); S. T. Chou, K. C. Hsieh, K. Y. Cheng, and L. J. Chou, J. Vac. Sci. Technol. B 13, 650 (1995).

2 J. Mirecki Millunchick, R. D. Twesten, D. M. Follstaedt, S. R. Lee, E. D. Jones, Y. Zhang, S. P. Ahrenkiel, and A. Mascarenhas, Appl. Phys. Lett. 70, 1402 (1997).

3 A. G. Norman, S. P. Ahrenkiel, H. Moutinho, C. Ballif, M. M. Al-Jassim, A. Mascarenhas, D. M. Follstaedt, S. R. Lee, J. L. Reno, E. D. Jones, J. Mirecki Millunchick, and R. D. Twesten, Mater. Res. Soc. Symp. Proc. 583, 297 (2000).

4 A. G. Norman, H. R. Moutinho, M. M. Al-Jassim, A. Mascarenhas, S. R. Lee, J. Mirecki Millunchick, J. L. Reno, and D. M. Follstaedt, Appl. Phys. Lett. (to be published).

5 A. Ponchet, A. Rocher, A. Ougazzaden, and A. Mircea, J. Appl. Phys. 75, 7881 (1994).

6 J. Tersoff, C. Teichert, and M. G. Lagally, Phys. Rev. Lett. 76, 1675 (1996); F. Liu, S. E. Davenport, H. M. Evans, and M. G. Lagally, ibid. 82, 2528 (1999).

7 L. E. Shilkrot, D. J. Srolovitz, and J. Tersoff, Appl. Phys. Lett. 77, 304 (2000); Phys. Rev. B 62, 8397 (2000).

8 Z. F. Huang and R. C. Desai, Phys. Rev. B 67, 075416 (2003).

9 J. Tersoff, Phys. Rev. B 43, 9377 (1991); B. J. Spencer, ibid. 59, 2011 (1999).

10 H. R. Eisenberg and D. Kandel, Phys. Rev. Lett. 85, 1286 (2000); Phys. Rev. B 66, 155429 (2002). 

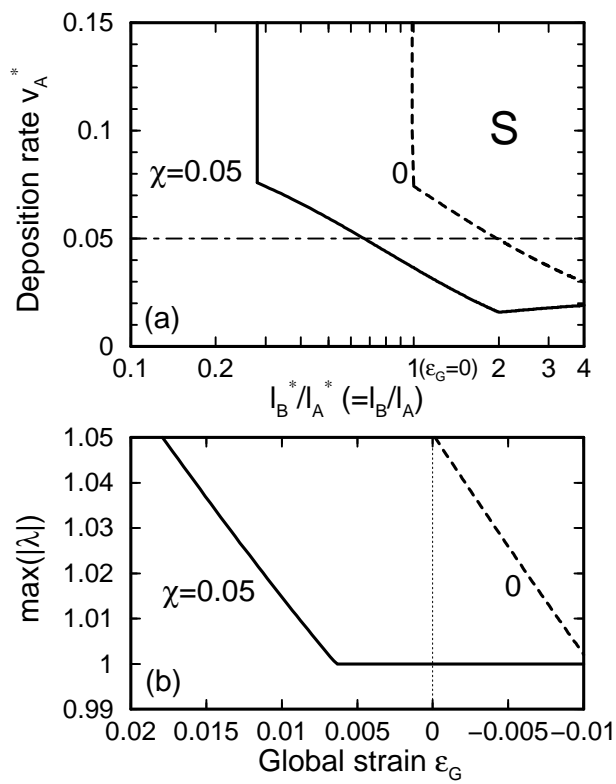

FIG. 1: (a) Stability diagram of $l_{B}^{*} / l_{A}^{*}$ vs rescaled deposition rate $v_{A}^{*}$, with $(\chi=0.05$, solid line $)$ or without $(\chi=0$, dashed line) the wetting effect. The parameters are analogous to those of AlAs/InAs on $\operatorname{InP}(001)$ superlattices growth: $\Gamma_{B}^{*}=0.001$, $l_{A}^{*}+l_{B}^{*}=0.3, \epsilon_{B}^{*}=-1, v_{B}^{*} / v_{A}^{*}=1$, and $\gamma_{B}^{*}=1$. (b) The maximum value of $|\lambda|$ as a function of multilayer global strain $\epsilon_{G}$, with the same parameters of (a) except for a fixed deposition rate $v_{A}^{*}=0.05$, corresponding to the horizontal dot-dashed line in (a).

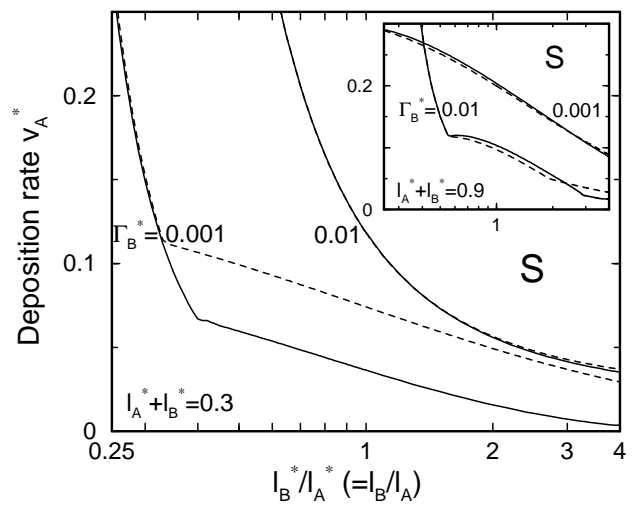

FIG. 2: Stability diagram of $l_{B}^{*} / l_{A}^{*}$ vs $v_{A}^{*}$ in the strain-balanced condition, with $(\chi=0.05$, solid line $)$ or without $(\chi=0$, dashed line) the wetting effect. The effects of different relative surface mobilities $\left(\Gamma_{B}^{*}=0.01\right.$ and 0.001$)$ and multilayer periods $\left[l_{A}^{*}+l_{B}^{*}=0.3\right.$ and 0.9 (inset) $]$ are shown. Other parameters are $v_{B}^{*} / v_{A}^{*}=1$ and $\gamma_{B}^{*}=1$ as in Fig. 1] 